\title{
Performance of Equal Phase-Shift Search for One Iteration
}

\author{
1 \\ Dafa Lid2, Jianping Chen ${ }^{a}$, Xiangrong $\mathrm{Li}^{b}$, Hongtao Huang ${ }^{c}$, Xinxin $\mathrm{Li}^{d}$ \\ ${ }^{a}$ Dept of mathematical sciences, Tsinghua University, Beijing 100084 CHINA \\ ${ }^{b}$ Department of Mathematics, University of California, Irvine, CA 92697-3875, USA \\ ${ }^{c}$ Electrical Engineering and Computer Science Department \\ University of Michigan, Ann Arbor, MI 48109, USA \\ ${ }^{d}$ Dept. of computer science, Wayne State University, Detroit, MI 48202, USA \\ Abstract \\ Grover presented the phase-shift search by replacing the selective inversions \\ by selective phase shifts of $\pi / 3$. In this paper, we investigate the phase-shift \\ search with general equal phase shifts. We show that for small uncertainties, the \\ failure probability of the Phase- $\pi / 3$ search is smaller than the general phase- \\ shift search and for large uncertainties, the success probability of the large \\ phase-shift search is larger than the Phase- $\pi / 3$ search. Therefore, the large \\ phase-shift search is suitable for large-size of databases.
}

PACS number: 03.67.Lx

Keywords: Amplitude amplification, the phase-shift search, quantum computing.

\section{Introduction}

Grover's quantum search algorithm is used to find a target state in an unsorted database of size $N[1]$ [2]. Grover's quantum search algorithm can be considered as a rotation of the state vectors in two-dimensional Hilbert space generated by the initial $(s)$ and target $(t)$ vectors [1. The amplitude of the desired state increases monotonically towards its maximum and decreases monotonically after reaching the maximum [3. As mentioned in 2] [4, unless we stop when it is right at the target state, it will drift away. A new search algorithm was presented in 2. to avoid drifting away from the target state. Grover proposed the new algorithm by replacing the selective inversions by selective phase shifts of $\pi / 3$, the algorithm converges to the target state irrespective of the number of iterations. In his paper, Grover demonstrated the power of his algorithm by calculating its success probability when only a single query into the database was allowed. It turned out that if the success probability for a random item in the database was $1-\epsilon$, where $\epsilon$ is known to randomly lie somewhere in the range $\left(0, \epsilon_{0}\right)$, after a single quantum query into the database, Grover's new phase-shift algorithm was able to increase the success probability to $1-\epsilon_{0}^{3}$. This was shown to be superior to existing algorithms and later shown to be optimal 7,8 .

In [5] 6], adiabatic quantum computation provides an alternative scheme for amplitude amplification that also does not drift away from the solution. In [8],

\footnotetext{
${ }^{1}$ The paper was supported by NSFC(Grants No. 60433050 and 60673034$)$, the basic research fund of Tsinghua university NO: JC2003043.

2email address:dli@math.tsinghua.edu.cn
} 
an algorithm for obtaining fixed points in iterative quantum transformations was presented and the average number of oracle queries for the fixed -point search algorithm was discussed. In [9], Boyer et al. described an algorithm that succeeds with probability approaching to 1. In [10, we discussed the phase-shift search algorithm with different phase shifts.

As discussed below, the implementation of the general phase-shift search relies on selective phase shifts. In this paper, we investigate the phase-shift search with general but equal phase shifts. We are able to considerably improve the algorithm by varying the phase-shift away from $\pi / 3$ when $\epsilon$ is large. As well known, the smaller deviation makes the algorithm converge to the target state more rapidly. The deviation for the Phase- $\pi / 3$ search is $\epsilon^{3}[2]$. For the large size of database, we investigate that the deviation for any phase shifts of $\theta>\pi / 3$ is smaller than $\epsilon^{3}$ and the closer to $\pi$ the phase shifts are, the smaller the deviation is. In this paper, we study the performance of the general phase-shift search for only one iteration. This also determines the failure probability and success probability of the general phase-shift search after recursively applying the single iteration for $n$ times. Note that we neglect the effects of decoherence completely in this paper.

This paper is organized as follows. In section 3, we give the necessary and sufficient conditions for the smaller deviation than $\epsilon^{3}$. In section 4 , we show that the Phase- $\pi / 3$ search algorithm performs well for the small $\epsilon$. In section 6 , we demonstrate that the closer to $\pi$ the phase shifts are, the smaller the deviation is. In section 7 , we propose the ratio measurement of the behavior of the Phase- $\theta$ search algorithm for one query.

\section{Grover's phase-shift search and the reduction of the deviation}

The standard amplitude amplification algorithm would overshoot the target state. To avoid drifting away from the target state, Grover presented the phaseshift search [2].

In [2] the transformation $U R_{s}^{\pi / 3} U^{+} R_{t}^{\pi / 3} U$ was applied to the initial state $|s\rangle$,

$$
\begin{aligned}
& R_{s}^{\pi / 3}=I-\left[1-e^{i \frac{\pi}{3}}\right]|s\rangle\langle s|, \\
& R_{t}^{\pi / 3}=I-\left[1-e^{i \frac{\pi}{3}}\right]|t\rangle\langle t|,
\end{aligned}
$$

where $|t\rangle$ stands for the target state. The transformation $U R_{s}^{\pi / 3} U^{+} R_{t}^{\pi / 3} U$ is denoted as Grover's the Phase- $\pi / 3$ search algorithm in 8 .

Grover let $\theta$ denote $\pi / 3$. Then

$$
\begin{aligned}
& R_{s}^{\theta}=I-\left[1-e^{i \theta}\right]|s\rangle\langle s|, \\
& R_{t}^{\theta}=I-\left[1-e^{i \theta}\right]|t\rangle\langle t| .
\end{aligned}
$$


The transformation $U R_{s}^{\theta} U^{+} R_{t}^{\theta} U$ is called as the Phase- $\theta$ search algorithm in this paper. As indicated in [2], when $\theta=\pi$, this becomes one iteration of the amplitude amplification algorithm[1] [4]. Note that if we apply $U$ to the initial state $|s\rangle$, then the amplitude of reaching the target state $|t\rangle$ is $U_{t s}[1$. Applying the transformation $U R_{s}^{\theta} U^{+} R_{t}^{\theta} U$ to the start state $|s\rangle$, Grover derived the following,

$$
U R_{s}^{\theta} U^{+} R_{t}^{\theta} U|s\rangle=U|s\rangle\left[e^{i \theta}+\left|U_{t s}\right|^{2}\left(e^{i \theta}-1\right)^{2}\right]+|t\rangle U_{t s}\left(e^{i \theta}-1\right) .
$$

Let $D(\theta)$ be the deviation from the $t$ state for any phase shifts of $\theta$. Then from (3) the following was obtained in [2],

$$
D(\theta)=\left.\left.\left(1-\left|U_{t s}\right|^{2}\right)\left|e^{i \theta}+\right| U_{t s}\right|^{2}\left(e^{i \theta}-1\right)^{2}\right|^{2} .
$$

Grover chose $\pi / 3$ as phase shifts and let $\left|U_{t s}\right|^{2}=1-\epsilon$, where $0<\epsilon<1$. Substituting $\left|U_{t s}\right|^{2}=1-\epsilon$, the deviation from the $t$ state becomes $D(\pi / 3)=$ $\epsilon^{3}[2]$.

Deviation $D(\theta)$ in (4) can be reduced as follows. For any $\theta$,

$$
e^{i \theta}+\left|U_{t s}\right|^{2}\left(e^{i \theta}-1\right)^{2}=e^{i \theta}+2(\cos \theta-1) e^{i \theta}(1-\epsilon)=e^{i \theta}[1+2(\cos \theta-1)(1-\epsilon)] .
$$

So by (15), we obtain

$$
D(\theta)=\epsilon[1+2(\cos \theta-1)(1-\epsilon)]^{2} .
$$

In this paper, we study the phase-shift search algorithm with two equal phase shifts. It is clear that it is enough to consider $\theta$ in $[0, \pi]$. It can be shown that the maximum and minimum of deviation $D(\theta)$ are 1 and 0 . That is,

$$
0 \leq D(\theta) \leq 1
$$

\section{The phase shifts for smaller deviation}

As indicated in [1, in the case of database search, $\left|U_{t s}\right|$ is almost $1 / \sqrt{N}$, where $N$ is the size of the database. Thus, $\epsilon$ is almost $1-1 / N$ and $\epsilon$ is close to 1 for the large size of database. It is known that the deviation for Grover's the Phase- $\pi / 3$ search is $\epsilon^{3}$. In this section, we give the phase shifts for smaller deviation than $\epsilon^{3}$.

\subsection{Necessary and sufficient conditions}

From (6) let us calculate

$$
\begin{aligned}
D(\theta)-\epsilon^{3} & =\epsilon[1+2(\cos \theta-1)(1-\epsilon)]^{2}-\epsilon^{3} \\
& =\epsilon(1-\epsilon)(2 \cos \theta-1)[2+(2 \cos \theta-3)(1-\epsilon)] .
\end{aligned}
$$

See Figs. 1 and 4. From (8), we have the following statement. 
Lemma 1. Deviation $D(\theta)$ in (6) for any phase shifts of $\theta$ in $[0, \pi / 3)$ is greater than $\epsilon^{3}$ for any $\epsilon$. That is, $D(\theta)>\epsilon^{3}$ for any $\theta$ in $[0, \pi / 3)$ and for any $\epsilon$. See table 1 .

The argument is as follows.

When $0 \leq \theta<\pi / 3,0<2 \cos \theta-1 \leq 1$ and $2 \epsilon<2+(2 \cos \theta-3)(1-\epsilon) \leq 1+\epsilon$ for any $\epsilon$. Therefore when $0 \leq \theta<\pi / 3$, it follows (8) that $D(\theta)>\epsilon^{3}$ for any $\epsilon$.

From (8) and Lemma 1, the following lemma holds immediately. See table 1.

Lemma 2. $D(\theta)<\epsilon^{3}$ if and only if

$$
\theta>\pi / 3 \wedge \epsilon>1-2 /(3-2 \cos \theta) .
$$

The following remark is used to describe the monotonicity of $1-2 /(3-2 \cos \theta)$ in (9). The monotonicity is used to find smaller deviation than $\epsilon^{3}$ below.

Remark $1.1-2 /(3-2 \cos \theta)$ increases from -1 to $3 / 5$ as $\theta$ increases from 0 to $\pi$. Thus,

$$
-1 \leq 1-2 /(3-2 \cos \theta) \leq 3 / 5 .
$$

Table 1. The phase shifts for deviations

\begin{tabular}{|c|c|c|}
\hline$\theta$ & & $\epsilon$ \\
\hline When $\theta>\pi / 3$ & $D(\theta)<\epsilon^{3}$ & for $\epsilon>1-2 /(3-2 \cos \theta)$ \\
\hline When $\theta<\pi / 3$ & $D(\theta)>\epsilon^{3}$ & for any $\epsilon$ \\
\hline When $\theta>\pi / 3$ & $D(\theta)>\epsilon^{3}$ & for $\epsilon<1-2 /(3-2 \cos \theta)$ \\
\hline
\end{tabular}

\subsection{The phase shifts for smaller deviation}

In this subsection, we give the phase shifts for which the deviations are smaller than $\epsilon^{3}$.

Corollary 1. Deviation $D(\theta)$ for any phase shifts of $\theta$ in $(\pi / 3, \alpha]$ is smaller than $\epsilon^{3}$ whenever $\epsilon>1-2 /(3-2 \cos \alpha)$.

Proof. By Remark $1,1-2 /(3-2 \cos \theta)$ increases from 0 to $1-2 /(3-2 \cos \alpha)$ as $\theta$ increases from $\pi / 3$ to $\alpha$. Thus, $0<1-2 /(3-2 \cos \theta) \leq 1-2 /(3-2 \cos \alpha)$ whenever $\pi / 3<\theta \leq \alpha$. Therefore, when $\epsilon>1-2 /(3-2 \cos \alpha)$, always $\epsilon$ $>1-2 /(3-2 \cos \theta)$. Hence, this corollary follows Lemma 2 .

When $\alpha=\pi, 2 \pi / 3, \pi / 2$ and $\arccos \frac{1-3 \delta}{2(1-\delta)}$, from Corollary 1 we have the following phase shifts for smaller deviations than $\epsilon^{3}$. See table 2 .

Result 1 . For any phase shifts of $\theta>\pi / 3$, deviation $D(\theta)<\epsilon^{3}$ for $\epsilon>3 / 5$. See Fig. 2 (a).

Result 2. For any phase shifts of $\theta$ in $(\pi / 3,2 \pi / 3]$, deviation $D(\theta)<\epsilon^{3}$ for $\epsilon>1 / 2$. See Fig. 2 (b).

Result 3. For any phase shifts of $\theta$ in $(\pi / 3, \pi / 2]$, deviation $D(\theta)<\epsilon^{3}$ for $\epsilon>1 / 3$. See Fig. 2 (c).

Result 4. When $\epsilon>\delta$, for any phase shifts of $\theta$ in $\left(\pi / 3, \arccos \frac{1-3 \delta}{2(1-\delta)}\right]$, deviation $D(\theta)<\epsilon^{3}$. 
Note that $\lim _{\delta \rightarrow 0} \arccos \frac{1-3 \delta}{2(1-\delta)}=\pi / 3$.

Our conclusion is when we search large database, i.e., $\epsilon$ is large, for any phase shifts of $\theta>\pi / 3$ the deviation is smaller than $\epsilon^{3}$.

Table 2. The phase shifts for $D(\theta)<\epsilon^{3}$

\begin{tabular}{|c|c|c|}
\hline$\theta$ & & $\epsilon$ \\
\hline When $\theta>\pi / 3$ & $D(\theta)<\epsilon^{3}$ & for $\epsilon>3 / 5$ \\
\hline When $\pi / 3<\theta \leq 2 \pi / 3$ & $D(\theta)<\epsilon^{3}$ & for $\epsilon>1 / 2$ \\
\hline When $\pi / 3<\theta \leq \pi / 2$ & $D(\theta)<\epsilon^{3}$ & for $\epsilon>1 / 3$ \\
\hline When $\pi / 3<\theta \leq \arccos \frac{1-3 \delta}{2(1-\delta)}$ & $D(\theta)<\epsilon^{3}$ & for $\epsilon>\delta$ \\
\hline
\end{tabular}

\section{The Phase- $\pi / 3$ search is optimal for small un- certainties.}

As indicated in [1, the size of the database is very large, i.e., $\epsilon$ is large. However, it is interesting to investigate the performances of the Phase- $\pi / 3$ search and the Phase- $\theta$ search for small $\epsilon$.

\subsection{The Phase- $\pi / 3$ search possesses smaller failure proba- bility}

As said in 2, $\epsilon^{3}$ and $D(\theta)$ are the failure probabilities of the Phase- $\pi / 3$ search and the Phase- $\theta$ search, respectively. Let us consider the ratio of the two failure probabilities. It is easy to see that $\lim _{\epsilon \rightarrow 0} \epsilon^{3} / D(\theta)=0$ for any $\theta \neq \pi / 3$. That is, $\epsilon^{3}=o(D(\theta))$. In other words, $\epsilon^{3}$ is smaller than $D(\theta)$ for small $\epsilon$. It means that $\epsilon^{3}$ approaches 0 more rapidly than $D(\theta)$ as $\epsilon$ approaches 0 .

\subsection{The conditions under which the Phase- $\pi / 3$ search be- haves well}

Here, we discuss what $\epsilon$ satisfies $\epsilon^{3}<D(\theta)$. From (8) and Lemma 1, we have the following lemma.

Lemma 3. $D(\theta)>\epsilon^{3}$ if and only if $\theta>\pi / 3$ and $\epsilon<1-2 /(3-2 \cos \theta)$ or $0 \leq \theta<\pi / 3$. See table 1 .

The following corollary follows Lemma 3 .

Corollary 2. When $\pi / 3<\alpha \leq \theta$ and $\epsilon<1-2 /(3-2 \cos \alpha), D(\theta)>\epsilon^{3}$.

The argument is as follows. By Remark $1,1-2 /(3-2 \cos \theta)$ increases from $1-2 /(3-2 \cos \alpha)$ to $3 / 5$ as $\theta$ increases from $\alpha$ to $\pi$. Thus, when $\pi / 3<\alpha \leq \theta$, $1-2 /(3-2 \cos \alpha) \leq 1-2 /(3-2 \cos \theta)$. Consequently, this corollary holds by Lemma 3.

From Corollary 2 we have the following results.

Result 5. When $\theta \geq \pi / 2, D(\theta)>\epsilon^{3}$ for $\epsilon<1 / 3$.

Result 6 . When $\theta \geq 2 \pi / 3, D(\theta)>\epsilon^{3}$ for $\epsilon<1 / 2$.

Result 7. When $\theta \geq \arccos \frac{1-3 \delta}{2(1-\delta)}, D(\theta)>\epsilon^{3}$ for $\epsilon<\delta$. 
Our conclusion is that for small $\epsilon$, the search algorithm performs optimal for $\theta=\pi / 3$. By means of the performance the Phase- $\pi / 3$ search algorithm can be applied to quantum error corrections.

\section{Zero deviation and average zero deviation points}

\subsection{Zero deviation}

Let $d=1+2(\cos \theta-1)(1-\epsilon)$. Then, deviation $D(\theta)$ in (6) can be rewritten as $D(\theta)=\epsilon d^{2}$. Let $d=0$. Then we obtain $\cos \theta=1-\frac{1}{2(1-\epsilon)}$, where $0<\epsilon \leq \frac{3}{4}$

to make $\left|1-\frac{1}{2(1-\epsilon)}\right| \leq 1$. Conclusively, if $U_{t s}$ is given, that is, $\epsilon$ is fixed, then we choose $\theta=\arccos \left[1-\frac{1}{2(1-\epsilon)}\right]$, which is in $(\pi / 3, \pi]$, as phase shifts. $\arccos \left[1-\frac{1}{2(1-\epsilon)}\right]$ will obviously make the deviation vanish and is called as a zero deviation point. It means that one iteration will reach $t$ state with certainty if the zero deviation point is chosen as phase shifts . Note that $\lim _{\epsilon \rightarrow 0} \arccos [1-$ $\left.\frac{1}{2(1-\epsilon)}\right]=\pi / 3$. This says that $\pi / 3$ is the limit of the zero deviation points $\theta$ though it is not a zero deviation point.

\subsection{Average zero deviation points}

When $0<\epsilon \leq \frac{3}{4}, \arccos \left[1-\frac{1}{2(1-\epsilon)}\right]$ is called as a zero deviation point. Since $\epsilon$ is not given, the zero deviation point is unknown. However, if we know the range of $\epsilon$, then in terms of mean-value theorem for integrals, we can find the average value $\bar{\theta}$ of the zero deviation points $\theta$. Here, we assume that $\epsilon$ is uniformly distributed in the interval $(\beta, \alpha) \subseteq(0,3 / 4]$.

Let $\epsilon$ be in the range $(\beta, \alpha)$, where $(\beta, \alpha) \subseteq(0,3 / 4]$. Then we calculate the average value of $1-\frac{1}{2(1-\epsilon)}$ over the range $(\beta, \alpha)$ as follows.

$$
\frac{1}{\alpha-\beta} \int_{\beta}^{\alpha}\left[1-\frac{1}{2(1-\epsilon)}\right] d \epsilon=1+\frac{1}{2(\alpha-\beta)} \ln \frac{1-\alpha}{1-\beta} .
$$

It can be argued that $-1 \leq 1+\frac{1}{2(\alpha-\beta)} \ln \frac{1-\alpha}{1-\beta}<\frac{1}{2}$. Thus, it is reasonable to define

$$
\bar{\theta}=\arccos \left[1+\frac{1}{2(\alpha-\beta)} \ln \frac{1-\alpha}{1-\beta}\right] .
$$

$\bar{\theta}$ can be considered as the average value of the zero deviation points $\theta$ and is called as the average zero deviation point. It can be seen that $\pi / 3<\bar{\theta} \leq \pi$.

When $\bar{\theta}$ is chosen as phase shift, we obtain the following deviation

$$
D(\bar{\theta})=\epsilon\left(1+\frac{1-\epsilon}{\alpha-\beta} \ln \frac{1-\alpha}{1-\beta}\right)^{2} .
$$


Let us compute $D(\bar{\theta})-\epsilon^{3}$ as follows.

$$
D(\bar{\theta})-\epsilon^{3}=\epsilon\left(1+\frac{1}{\alpha-\beta} \ln \frac{1-\alpha}{1-\beta}\right)(1-\epsilon)\left(1+\frac{1-\epsilon}{\alpha-\beta} \ln \frac{1-\alpha}{1-\beta}+\epsilon\right) .
$$

Notice that $1+\frac{1}{\alpha-\beta} \ln \frac{1-\alpha}{1-\beta}<0$ and $1-\frac{1}{\alpha-\beta} \ln \frac{1-\alpha}{1-\beta}>0$. Let $\kappa=1-2 /(1-$ $\left.\frac{1}{\alpha-\beta} \ln \frac{1-\alpha}{1-\beta}\right)$. It can be proven that $0<\kappa<1$. We can conclude when $\epsilon>\kappa$, $D(\bar{\theta})<\epsilon^{3}$.

We will find the average zero deviation point $\bar{\theta}$ for the ranges $(0,1 / 2)$ and $(0,3 / 4)$ of $\epsilon$, respectively, as follows.

Example 1. Let $\epsilon$ lie in the range $(0,1 / 2]$. By (12), the average zero deviation point $\bar{\theta}_{1}=\arccos (1-\ln 2)=72^{\circ} 30^{\prime}$. Taking $\bar{\theta}_{1}$ as phase shifts, by (13) deviation $D\left(\bar{\theta}_{1}\right)=\epsilon[1-2(1-\epsilon) \ln 2]^{2}$. Deviation $D\left(\bar{\theta}_{1}\right)$ for phase shifts of $\bar{\theta}_{1}$ is smaller than $\epsilon^{3}$, i.e., $D\left(\bar{\theta}_{1}\right)<\epsilon^{3}$, if and only if $\epsilon>\frac{2 \ln 2-1}{2 \ln 2+1}=0.16$.

Example 2. Let $(0,3 / 4]$ be the range of $\epsilon$. Then by (12), the average zero deviation point $\bar{\theta}_{2}=\arccos \left(1-\frac{4}{3} \ln 2\right)=86^{\circ}$. Choosing $\bar{\theta}_{2}$ as phase shifts, by (13) deviation $D\left(\bar{\theta}_{2}\right)=\epsilon\left[1-\frac{8}{3}(1-\epsilon) \ln 2\right]^{2}$ and $D\left(\bar{\theta}_{2}\right)$ is smaller than $\epsilon^{3}$ when $\epsilon>0.30$.

\section{Monotonicity of the deviation for large $\epsilon$}

As discussed above, when $\epsilon$ is fixed and lies in the range $(0,3 / 4]$ and $\arccos (1-$ $\left.\frac{1}{2(1-\epsilon)}\right)$ is chosen as phase shifts, the deviation vanishes. When $\epsilon>3 / 4$, since $\left|1-\frac{1}{2(1-\epsilon)}\right|>1$, deviation $D(\theta)$ does not vanish for any phase shifts of $\theta$ in $[0, \pi]$.

When $\epsilon \geq \frac{3}{4}$,

$$
-1 \leq 2(\cos \theta-1)(1-\epsilon) \leq 0 .
$$

and $0 \leq d \leq 1$. When $U_{t s}$ is given, that is, $\epsilon$ is fixed, by using (15) it can be shown that deviation $D(\theta)$ monotonically decreases from $\epsilon$ to $\epsilon(4 \epsilon-3)^{2}$ as $\theta$ increases from 0 to $\pi$. See Fig. 3. for the monotonicity of $D(\theta)$. When $\theta=\pi$, the deviation gets its minimum $\epsilon(4 \epsilon-3)^{2}$. That is,

$$
\epsilon(4 \epsilon-3)^{2} \leq D(\theta)
$$

for any phase shifts of $\theta$ in $[0, \pi]$, whenever $\epsilon \geq 3 / 4$.

Peculiarly, the deviation $\epsilon(4 \epsilon-3)^{2}<\epsilon^{3}$ whenever $\epsilon>3 / 5$. The inequality in (16) also follows that $(4 \epsilon-3) \leq d$ for any phase shifts of $\theta$ in $[0, \pi]$ whenever $\epsilon \geq 3 / 4$.

See table 3 for the deviations $D(\theta)$ for $\theta=\pi / 2,2 \pi / 3,3 \pi / 4,5 \pi / 6, \pi$. Also see Fig. 4.

Table 3. The deviations for $\epsilon>3 / 4$

\begin{tabular}{|c|c|c|c|c|}
\hline$\theta$ & $\pi / 2$ & $2 \pi / 3$ & $3 \pi / 4$ & $5 \pi / 6$ \\
\hline$D(\theta)$ & $\epsilon(2 \epsilon-1)^{2}$ & $\epsilon(3 \epsilon-2)^{2}$ & $\epsilon((\sqrt{2}+2) \epsilon-(\sqrt{2}+1))^{2}$ & $\epsilon((\sqrt{3}+2) \epsilon-(\sqrt{3}+1))^{2}$ \\
\hline
\end{tabular}


Remark 2.

From the discussion above, it is easy to see that the closer to $\pi$ the phase shifts are, the smaller the deviation is, when $\epsilon \geq \frac{3}{4}$. By means of the inequality in (16) we can discuss the lower bound of the number of iterations to find the $t$ state.

Note that when the selective phase shift $\theta$ becomes $\pi$, the phase- $\pi$ search is the amplitude amplification search.

\section{The ratio measurement of the success proba- bilities for one query}

\subsection{The ratio of the success probabilities}

Clearly, the greater the success probability is, the better the algorithm performs. In other words, the more rapidly the algorithm converges. In this section, it is demonstrated that the limit of the ratio of success probabilities of the Phase- $\theta$ and the Phase- $\pi / 3$ search algorithms is used to quantify the performance of the Phase- $\theta$ search algorithm.

From (7), let $\Delta(\theta)=1-D(\theta)$. Then $\Delta(\theta)$ is the success probability with which the transformation $U R_{s}^{\theta} U^{+} R_{t}^{\theta} U$ in (3) drives the start state to the target state. For instance, $\Delta(\pi / 3)=1-D(\pi / 3)=1-\epsilon^{3}$, which is the success probability of the Phase- $\pi / 3$ search algorithm for one query. See Page 1 in [2]. Explicitly, $\Delta(\theta)$ is not the desired measurement free of $\epsilon$ for the Phase- $\theta$ search algorithm because $\Delta(\theta)$ is also a function of $\epsilon$.

Let us compute the limit of $\Delta(\theta)$ as $\epsilon$ approaches 1 as follows.

$\left.\lim _{\epsilon \rightarrow 1} \Delta(\theta)=\lim _{\epsilon \rightarrow 1}\left(1-\epsilon(1+2(\cos \theta-1)(1-\epsilon))^{2}\right)\right)=0$, for any $\theta$ in $[0, \pi]$.

It is straightforward that the above limit can not be used to describe the performance of the Phase- $\theta$ search algorithm for any phase shifts of $\theta$ in $[0, \pi]$ because the limit always is zero for any $\theta$ in $[0, \pi]$.

It is natural to consider and calculate $\frac{\Delta(\theta)}{\Delta(\pi / 3)}$ as follows.

$$
\frac{\Delta(\theta)}{\Delta(\pi / 3)}=\frac{4\left(\cos ^{2} \theta\right) \epsilon^{2}-8(\cos \theta) \epsilon^{2}+4 \epsilon^{2}+4(\cos \theta) \epsilon-4\left(\cos ^{2} \theta\right) \epsilon+1}{\epsilon^{2}+\epsilon+1} .
$$

Then we obtain the following limit of $\frac{\Delta(\theta)}{\Delta(\pi / 3)}$ as $\epsilon$ approaches 1. Let

$$
\rho=\lim _{\epsilon \rightarrow 1} \frac{\Delta(\theta)}{\Delta(\pi / 3)}=\frac{5-4 \cos \theta}{3} .
$$

Then $\rho$ can be considered as the ratio of success probabilities for the Phase- $\theta$ and the Phase- $\pi / 3$ search algorithms for large $\epsilon$. Notice that $\rho$ is free of $\epsilon$ and only depends on $\theta$. Hence, $\rho$ can be considered as a measurement of performance of Phase- $\theta$ search algorithm for any phase shifts of $\theta$ in $[0, \pi]$. 
We can follow [2] to define by the recursion $U_{m+1}=U_{m} R_{s}^{\theta} U_{m}^{+} R_{t}^{\theta} U_{m}$, where $U_{0}=U$. For the Phase- $\pi / 3$ search, after recursive application of the basic iteration $m$ times, the success probability $\left|U_{m, t s}\right|=1-\epsilon^{3^{m}}[2$. For the Phase$\theta$ search, as well we can derive the success probability $\left|U_{m, t s}\right|$ and the failure probability $1-\left|U_{m, t s}\right|$ after recursive application of the basic iteration $m$ times. Fixed points of the Phase- $\theta$ search algorithm are discussed in [11.

\subsection{The larger phase shifts than $\pi / 3$ for larger size of database}

It can be shown that $\rho$ increases from $1 / 3$ to 3 as $\theta$ increases from 0 to $\pi$. In particular, $\rho$ increases from 1 to 3 as $\theta$ increases from $\pi / 3$ to $\pi$. This also says that for large databases, the larger the phase shifts are, the greater the success probabilities are. For instance, $\rho=2.8$ for Phase- $5 \pi / 6$ search. This means that for large $\epsilon$, the ratio of success probabilities for the Phase- $5 \pi / 6$ and the Phase- $\pi / 3$ search is 2.8 . See table 4 .

Table 4. $\rho$ 's values for the Phase- $\theta$ search

\begin{tabular}{|c|c|c|c|c|c|}
\hline$\theta$ & $\pi / 2$ & $2 \pi / 3$ & $3 \pi / 4$ & $5 \pi / 6$ & $\pi$ \\
\hline$\rho$ & $5 / 3$ & $7 / 3$ & $(5+2 \sqrt{2}) / 3=2.6$ & $(5+2 \sqrt{3}) / 3=2.8$ & 3 \\
\hline
\end{tabular}

\section{Summary}

In this paper, we give the phase shifts for smaller deviation than $\epsilon^{3}$. When $\epsilon \leq 3 / 4$ and $\epsilon$ is given, we choose the zero deviation point as phase shifts to find the desired state for one iteration. When $\epsilon \geq 3 / 4$, the deviation decreases from $\epsilon^{3}$ to $\epsilon(4 \epsilon-3)^{2}$ as $\theta$ increases from $\pi / 3$ to $\pi$. It is shown that for small $\epsilon$, the Phase- $\pi / 3$ search behaves better than the general Phase- $\theta$ search. Therefore the Phase- $\pi / 3$ search can be applied to quantum error correction. We propose the limit of the ratio of success probabilities of the Phase- $\theta$ and the Phase- $\pi / 3$ search algorithms as a measure of efficiency of a single Phase- $\theta$ iteration. The measure can help us find the optimal phase shifts for small deviation and large success probability. Thus, there are more choices for phase shifts to adjust an algorithm for large size of database and more loose constraint opens a door for more feasible or robust realization.

Acknowledgement

We want to thank Lov K. Grover for his helpful discussions and comments on the original manuscript ( in December, 2005) and the reviewer for the helpful comments on this paper and useful discussions about fixed points of the Phase- $\theta$ search.

\section{References}

[1] L.K.Grover, Phys. Rev. Lett. 80, 4329 (1998).

[2] L.K.Grover, Phys. Rev. Lett. 95, 150501 (2005). 
[3] D. Li et al., Theoretical and Mathematical Physics, 144(3):1279$1287(2005)$.

[4] G. Brassard, Science 275, 627 (1997).

[5] E. Farhi et al., Science 292, 472, 2001.

[6] J. Roland and N.J. Cerf, Phys. Rev. A 65, 042308, 2002.

[7] L.K.Grover et al., quant-ph/0603132.

[8] T.Tulsi, L. Grover, and A. Patel, quant-ph/0505007, Also, Quantum Information and Computation 6(6), 483-494, 2006.

[9] M. Boyer et al., quant-ph/9605034, Fortschr. Phys. 46, 493 (1998).

[10] D. Li et al., Phys. Lett. A 362(2007) 260-264. Also see quant-ph/0604062.

[11] In the reviewer's report on this paper, the reviewer investigated fixed points of the Phase- $\theta$ search algorithm as follows. The reviewer let $f\left(\epsilon_{n}\right)=\epsilon_{n+1}=$ $\epsilon_{n}\left[1+2(\cos \theta-1)\left(1-\epsilon_{n}\right)\right]^{2}$. Then the reviewer showed that the equation has 0 to be an attractive fixed point when $0<\theta<\pi / 2$ because $0<f^{\prime}(0)<1$, $\cos \theta /(\cos \theta-1)$ to be an attractive fixed point when $\pi / 2<\theta<2 \pi / 3$ because $\left|f^{\prime}(\cos \theta /(\cos \theta-1))\right|<1$, and all the fixed points to be repulsive when $2 \pi / 3<\theta<\pi$. Following the reviewer's idea, we can show when $\theta=\pi / 2,0$ is a semi-attractive fixed point because $f^{\prime}(0)=1$ and $f^{\prime \prime}(0)=$ -8 and when $\theta=2 \pi / 3,1 / 3$ is also a semi-attractive fixed-point because $f^{\prime}(1 / 3)=-1$ and $f^{\prime \prime}(1 / 3)=-6$. 